\title{
Creation of Exclusive Artificial Cluster Defects by Selective Metal Removal in the (Zn, Zr) Mixed-metal UiO-66.
}

Xiao Feng*[a,b], Himanshu Sekhar Jena[b], Chidharth Krishnaraj[b], Daniel ArenasEsteban[c], Karen Leus[b], Guangbo Wang[b,d], Martina Rüscher[e], Janis Timoshenko[e], Beatriz Roldan Cuenya[e], Sara Bals[c] and Pascal Van Der Voort*[b]

[a]Dr. X. Feng, Zhang Dayu School of Chemistry, State Key Laboratory of Fine Chemicals, Dalian University of Technology, 116024 Dalian, China

[b] Dr. X. Feng, Dr. H. S. Jena, Dr. C. Krishnaraj, Dr. K. Leus, Dr. G. Wang, Prof. P. Van Der Voort, Center for Ordered Materials, Organometallics and Catalysis (COMOC), Department of Chemistry, Ghent University, 281, Krijgslaan (S3), B-9000, Ghent, Belgium

[c] Dr. Arenas-Esteban, Prof. Sara Bals, Dept. of Physics, EMAT - Electron Microscopy for Materials Science, University of Antwerp, Groenenborgerlaan 171, 2020 Antwerp, Belgium.

[d] Dr. G. Wang, Chemical Engineering and Materials Science, College of Chemistry, Shandong Normal University, Collaborative Innovation Center of Functionalized Probes for Chemical Imaging in Universities of Shandong, Key Laboratory of Molecular and Nano Probes, Ministry for Education, Jinan 250014, P. R. China.

[e] MSc. Martina Rüscher, Dr. Janis Timoshenko, Prof. Beatriz Roldan Cuenya, Interface Science Department, Fritz-Haber Institute of the Max-Planck Societey, Faradayweg 4-6, 14195 Berlin, Germany.

\section{General Procedures}

All chemicals were purchased from Sigma Aldrich, ABCR or TCI Europe and used without further purification. X-ray powder diffraction (PXRD) patterns were collected on an ARL X'TRA X-ray diffractometer operating at $40 \mathrm{kV} / 40 \mathrm{~mA}$ using $\mathrm{Cu}-\mathrm{K} \alpha$ radiation $(\lambda=0.15418 \mathrm{~nm})$ and a solid-state detector. Nitrogen adsorption experiments were carried out at $77 \mathrm{~K}$ using a Belsorp-mini II gas analyzer. Prior to adsorption measurements, the samples were activated under vacuum at $120^{\circ} \mathrm{C}$ for $16 \mathrm{~h}$ to remove adsorbed solvent. Thermogravimetric analysis was performed on a Netzsch STA 449 F3 Jupiter with a temperature range of $20-800{ }^{\circ} \mathrm{C}$ in air with a heating rate of $2{ }^{\circ} \mathrm{C} / \mathrm{min}$. An ultra-fast $\mathrm{GC}$ equipped with a flame ionization detector (FID) and a 5\% diphenyl/95\% polydimethylsiloxane column, with $10 \mathrm{~m}$ length and $0.10 \mathrm{~mm}$ internal diameter was used to follow the conversions of the products during the catalytic tests. Helium was used as carrier gas and the flow rate was programmed as $0.6 \mathrm{~mL} / \mathrm{min}$. The reaction products were identified with a TRACE GC $\times$ GC (Thermo, Interscience), coupled to a TEMPUS TOF-MS detector (Thermo, Interscience). The first column consists of a dimethyl polysiloxane package and has a length of $50 \mathrm{~m}$, with an internal diameter of $0.25 \mathrm{~mm}$, whereas the second column has a length of $2 \mathrm{~m}$ with an internal diameter of $0.15 \mathrm{~mm}$. 
The package of the latter is a $50 \%$ phenyl polysilphenylene-siloxane. $\mathrm{CO}_{2}$ and $\mathrm{N}_{2}$ adsorption measurements were carried out on a Quantachrome iSorb-HP gas sorption analyzer.

\section{Experimental section}

\section{Synthesis of UiO-66 with artificial cluster defects}

The bimetallic (Zn, Zr)-UiO-66 was synthesized according to the procedure of Shearer et al. ${ }^{1} 1.891 \mathrm{~g} \mathrm{ZrCl}_{4}$ $(8.11 \mathrm{mmol})$ and the corresponding equivalents of $\mathrm{Zn}\left(\mathrm{NO}_{3}\right)_{2} \cdot 6 \mathrm{H}_{2} \mathrm{O}(\mathrm{Zn}: \mathrm{Zr}=1: 10,1: 3,2: 3,1: 1)$ were dissolved in $48.7 \mathrm{~mL} \mathrm{~N}$,N-dimethyl formamide (DMF). 2.696g terephthalic acid $\left(\mathrm{H}_{2} \mathrm{BDC}, 16.22 \mathrm{mmol}\right)$ and $1.433 \mathrm{~mL}$ $\mathrm{HCl}(16.22 \mathrm{mmol})$ were added. The mixture was sonicated for $30 \mathrm{~min}$ and then transferred into a Teflon-lined autoclave and heated to $220^{\circ} \mathrm{C}$ for $24 \mathrm{~h}$. After the autoclave reached room temperature, the white solids that formed were collected by means of filtration, and washed extensively with DMF and methanol. In a following step, the obtained bimetallic UiO-66 with different ratios of $\mathrm{Zn}$ and $\mathrm{Zr}$ were stirred for $10 \mathrm{~min}$ in $\mathrm{HCl}(\mathrm{pH}=$ 1), filtered; and washed with water until $\mathrm{pH}$ neutral, and finally washed with methanol. The solids were then soaked in methanol for $24 \mathrm{~h}$ and dried at $65^{\circ} \mathrm{C}$ under vacuum.

\section{Catalytic reaction}

The ring-opening reactions of styrene oxide with isopropanol were carried out in a $1 \mathrm{~mL}$ vial. A mixture of styrene oxide ( $23 \mu \mathrm{L}, 0.2 \mathrm{mmol})$, anhydrous isopropanol ( $500 \mu \mathrm{L}, 6.5 \mathrm{mmol})$, and dodecane (45 $\mu \mathrm{L}, 0.2 \mathrm{mmol})$

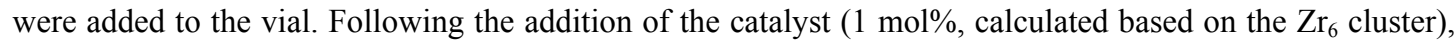
the vial was sealed and put into an oil bath (temperature: $55^{\circ} \mathrm{C}$, agitation speed: $550 \mathrm{rpm}$ ). The reaction products were analysed, after a reaction time of $24 \mathrm{~h}$, by means of GC and were confirmed by GC-MS.

\section{iDPC-STEM characterization}

The iDPC-STEM images were acquired using a Thermo Fisher Scientific Titan Cubed transmission electron microscope. This instrument is equipped with a D-Corr probe aberration corrector and was operated at $300 \mathrm{kV}$ with a convergence semi-angle of $10.8 \mathrm{mrad}$. Four images used for $2 \mathrm{D}$ integration were acquired by a fourquadrant DF4 detector with an optional high-pass filter of sigma=80 applied to reduce the low-frequency information in the image. The electron-beam current was reduced lower than $1.5 \mathrm{pA}$. The collection angles of iDPC-STEM were set as $5 \sim 19 \mathrm{mrad}$. The dwell time of the image is $4 \mu$ s and the pixel size $35.6 \mathrm{pm}$ resulting in an electron dose of $<300 \mathrm{e}^{-/} \AA^{2}$.

\section{XAS Experimental}

For XAS measurements, samples in powder form (60-120 mg, dependent on the $\mathrm{Zn}$ amount) were mixed with boron nitride (ca $150 \mathrm{mg}$ ) and pressed into $13 \mathrm{~mm}$ pellets. XAS measurements at $\mathrm{Zn} \mathrm{K}$-edge (9659 eV) and $\mathrm{Zr}$ K-edge $(17998 \mathrm{eV})$ were performed at CLAESS beamline at ALBA synchrotron radiation facility (Barcelona). Measurements were performed in transmission and fluorescence mode. At Zr K-edge, transmission XAS data were used for further analysis. At Zn K-edge, transmission data were used only for the edge step determination (to estimate $\mathrm{Zn}$ to $\mathrm{Zr}$ ratio), while for the further analysis we relied on the data collected in fluorescence mode.

Intensities of the incident radiation and transmitted radiation were measured with ionization chamber detectors $I_{0}$ and $I_{1}$ filled with pure $\mathrm{N}_{2}\left(I_{0}\right.$ chamber) or 70:30 $\mathrm{N}_{2}$ and $\mathrm{Kr}$ mixture $\left(I_{1}\right.$ chamber). Fluorescence data were collected using energy selective 6-channel Si drift detector. Si (111) monochromator was used for energy selection. For data alignment, XAS spectra for $\mathrm{Zn}$ and $\mathrm{Zr}$ foil XAS spectra were acquired in transmission mode simultaneously with the spectra for MOF samples. Spectrum for $\mathrm{ZnO}$ pellet was also acquired as a reference. 
Reference spectrum for $\mathrm{ZrO}_{2}$ was taken from Ref ${ }^{2}$. All measurements were performed in air at room temperature.

\section{XAS Data analysis}

ATHENA software ${ }^{3}$ was used for data alignment, normalization and extraction of X-ray absorption near edge structure (XANES) and extended X-ray absorption fine structure (EXAFS) spectra. For quantitative EXAFS analysis we performed non-linear least square fitting to theoretical standards, as implemented in FEFFIT code. ${ }^{3}$ Theoretical phases and amplitudes were obtained in self-consistent ab-initio calculations with FEFF8.5 code ${ }^{4}$ for reference MOF structure from Ref. ${ }^{5}$ The complex exchange-correlation Hedin-Lundqvist potential and default values of muffin-tin radii as provided within the FEFF8.5 code were employed. Fitting of EXAFS spectra $\chi(\mathrm{k}) \mathrm{k}^{2}$ was carried out in $R$-space in the range from $R_{\min }=1.0 \AA$ up to $R_{\max }$, where $R_{\max }$ was set to 3.5 $\AA$ for $\mathrm{Zr} \mathrm{K}$-edge data, and to $2.5 \AA$ for Zn K-edge data, thus including the contributions of the first two, or only the first coordination shell, respectively. At Zr K-edge Fourier transform was carried out in the $k$-range from $3.0 \AA^{-1}$ up to $15 \AA^{-1}$. At Zn K-edge Fourier transform was carried out in the $k$-range from $3.0 \AA^{-1}$ up to $10 \AA^{-1}$ (for samples S1 and S2), and up to $8 \AA^{-1}$ (for sample S3). Fitting parameters were coordination numbers $N$, interatomic distances $R$ and disorder factors $\sigma^{2}$ corresponding to $\mathrm{Zr}-\mathrm{O}, \mathrm{Zr}-\mathrm{Zr}, \mathrm{Zr}-\mathrm{Zn}$ and $\mathrm{Zn}-\mathrm{O}$ bonds. We have included third cumulant for Zn-O bond when fitting Zn K-edge EXAFS, to account for significantly distorted and disordered local environment of $\mathrm{Zn}$ species. In addition, correction to photoelectron reference energy $\Delta E_{0}$ was treated as a fitting parameter. Amplitude reduction factor $S_{0}^{2}$ was set to 1.0, as estimated from the fitting of EXAFS spectra for reference materials. 


\section{Supporting Figures}
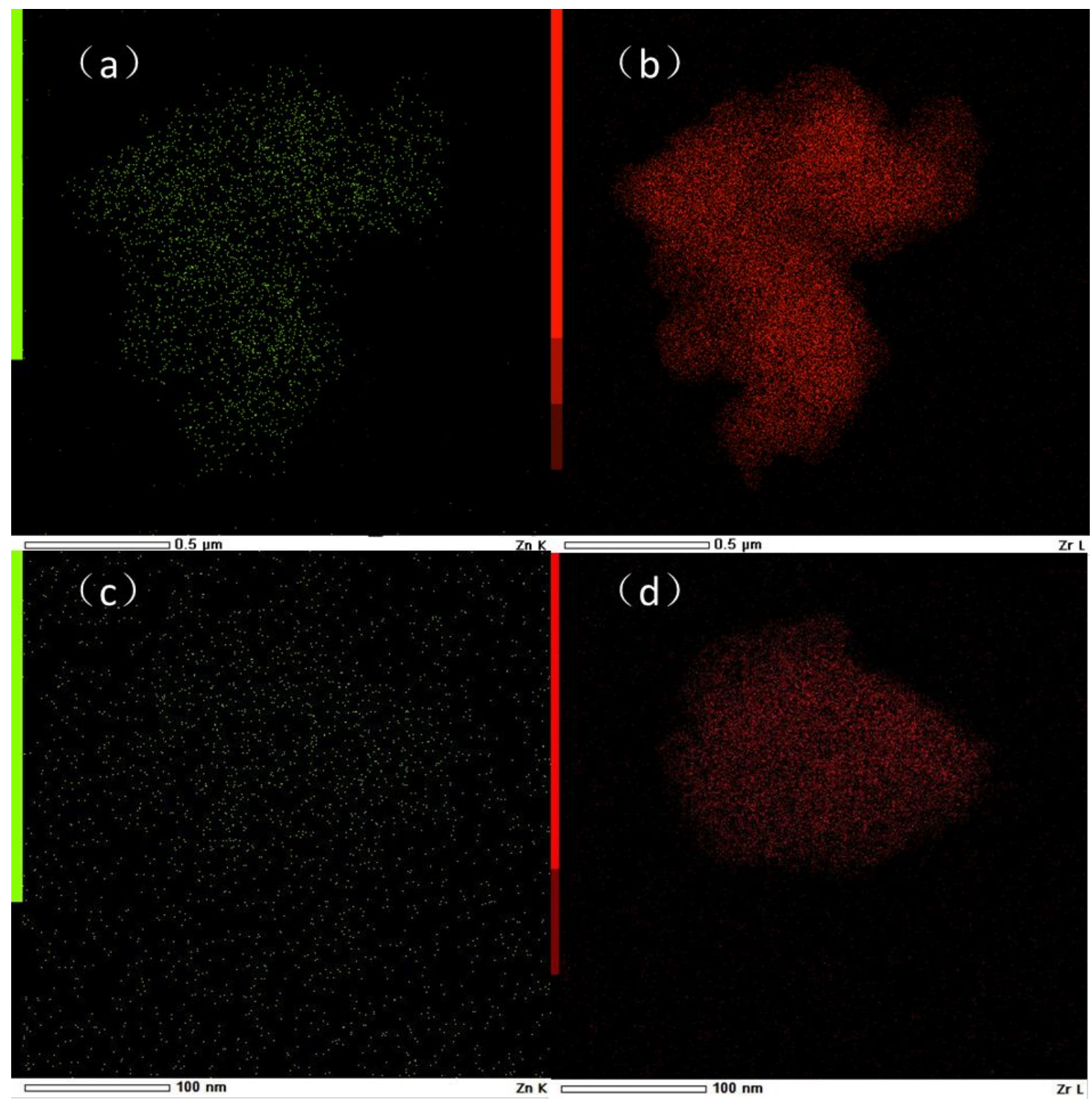

Figure S1. EDS-mapping analysis of UiO-66-0.33 before acid washing (a. Zn b. Zr) and after acid washing (c. Zn d. $\mathrm{Zr}$ ). 

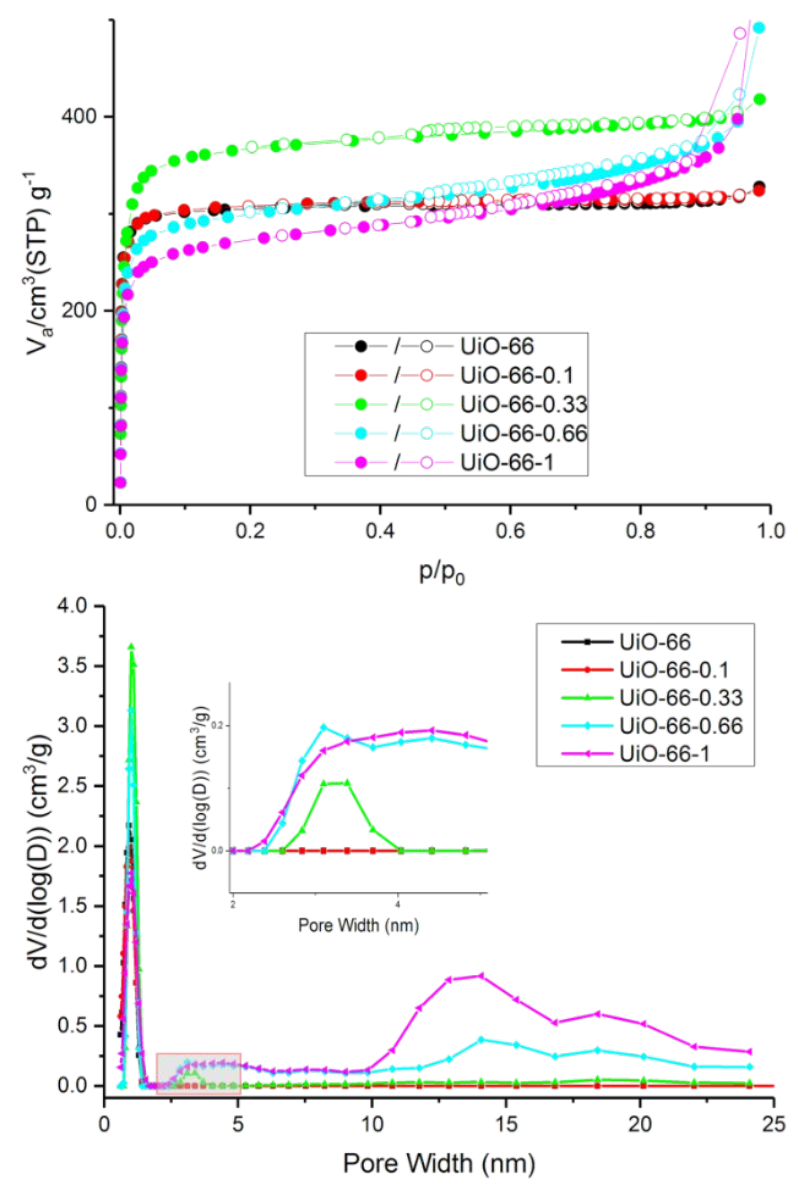

Figure. S2 Nitrogen adsorption isotherms of the UiO-66 samples obtained using different ratios of $\mathrm{Zr}$ and $\mathrm{Zn}$ (top). Pore size distribution of the UiO-66 samples obtained using different ratios of $\mathrm{Zr}$ and $\mathrm{Zn}$ according to non-local density functional theory (bottom).
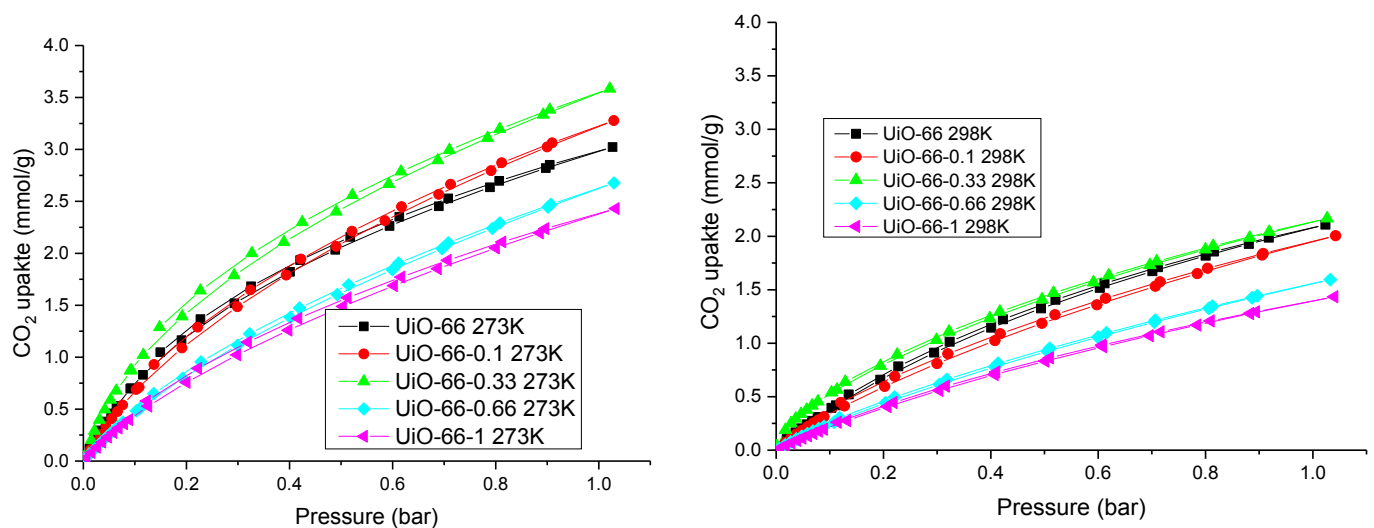

Fig. S3 $\mathrm{CO}_{2}$ adsorption and desorption isotherms of UiO-66 samples obtained using different ratios of $\mathrm{Zr}$ and $\mathrm{Zn}$ at $273 \mathrm{~K}$ and $298 \mathrm{~K}$. 


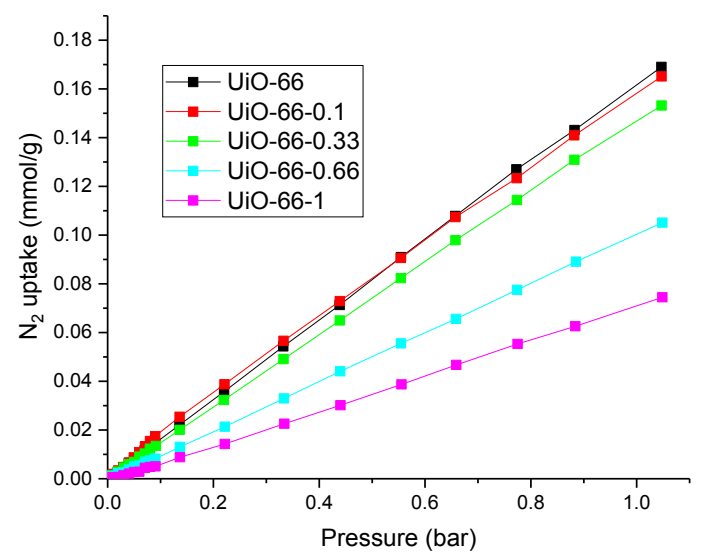

Fig. S4 $\mathrm{N}_{2}$ adsorption isotherms of UiO-66 samples obtained using different ratios of $\mathrm{Zr}$ and $\mathrm{Zn}$ at $298 \mathrm{~K}$.

\section{EXAFS data}

Extracted EXAFS spectra and their Fourier transforms (FT) for MOF samples and reference oxides are shown in Figure 6 and Figure S6, respectively.

Zr K-edge FT-EXAFS spectra (Figure S6b) are similar to those reported for Zr K-edge in (Ce, Zr) UiO-66 MOF, ${ }^{6}$ and are dominated by the two peaks, one at the ca. $1.5 \AA$, another at ca. $3.2 \AA$. The first peak we attribute to the contribution of $\mathrm{Zr}-\mathrm{O}$ bonds, and its position and intensity are similar to those for $\mathrm{Zr}-\mathrm{O}$ peak in $\mathrm{ZrO}_{2}$. The second peak we tentatively attribute to $\mathrm{Zr}-\mathrm{M}$ bond (here $\mathrm{M}$ is $\mathrm{Zn}$ or $\mathrm{Zr}$ ). This peak in all MOF samples is shifted to larger interatomic distances than $\mathrm{Zr}-\mathrm{Zr}$ peak in $\mathrm{ZrO}_{2}$. Moreover, we observe systematic decrease in the intensity of this feature, when going from sample S1 to S2, to S3. The peak position, nevertheless, remain the same. This may be already and indication that in all cases this peak corresponds to the same type of bond (i.e., $\mathrm{Zr}-\mathrm{Zr}$ ), and the contribution of heterometallic $\mathrm{Zr}-\mathrm{Zn}$ bonds is not significant.

At $\mathrm{Zn} \mathrm{K-edge,} \mathrm{we} \mathrm{observe} \mathrm{that} \mathrm{the} \mathrm{mismatch} \mathrm{between} \mathrm{the} \mathrm{EXAFS} \mathrm{frequencies} \mathrm{for} \mathrm{MOF} \mathrm{sample} \mathrm{and} \mathrm{ZnO}$ reference is more pronounced (Figure 6d), indicating the difference in $\mathrm{Zn}-\mathrm{O}$ bond lengths (in agreement with the observations from XANES data). Moreover, we observe significant difference in EXAFS frequencies for MOF samples S1 and S2, and MOF sample S3. In the latter case, the first EXAFS features resemble those in $\mathrm{ZnO}$, but with suppressed amplitude, suggesting that the remaining $\mathrm{Zn}$ species may be present in this MOF sample in the form of disordered oxide clusters. Unlike it was in the case of Zr K-edge EXAFS, at Zn K-edge Fourier transformed EXAFS is dominated by a single peak at ca. 1.5-2 $\AA$, which corresponds to Zn-O bonds. In the pristine sample $\mathrm{S} 1$, this peak is noticeably shifted to larger interatomic distances in comparison to that in $\mathrm{ZnO}$ reference. Upon washing treatment, however, this peak shifts to shorter interatomic distances, and in the sample $\mathrm{S} 3$ it is at a position roughly corresponding to the $\mathrm{Zn}-\mathrm{O}$ peak position in $\mathrm{ZnO}$. The shift of $\mathrm{Zn}-\mathrm{O}$ peak is paralleled by its broadening, which reveals a significant increase in the structural disorder around $\mathrm{Zn}$ species. Crucially, the contribution of distant coordination shells is relatively weak in Zn K-edge EXAFS for MOF samples, and only a small broad peak is present at $R$ values ca. 3-4 A. For S3 sample, only the first peak can be identified reliably: the other peaks in FT-EXAFS for this sample are artifacts due to very low Zn loading and, consequently, poor EXAFS data quality. 

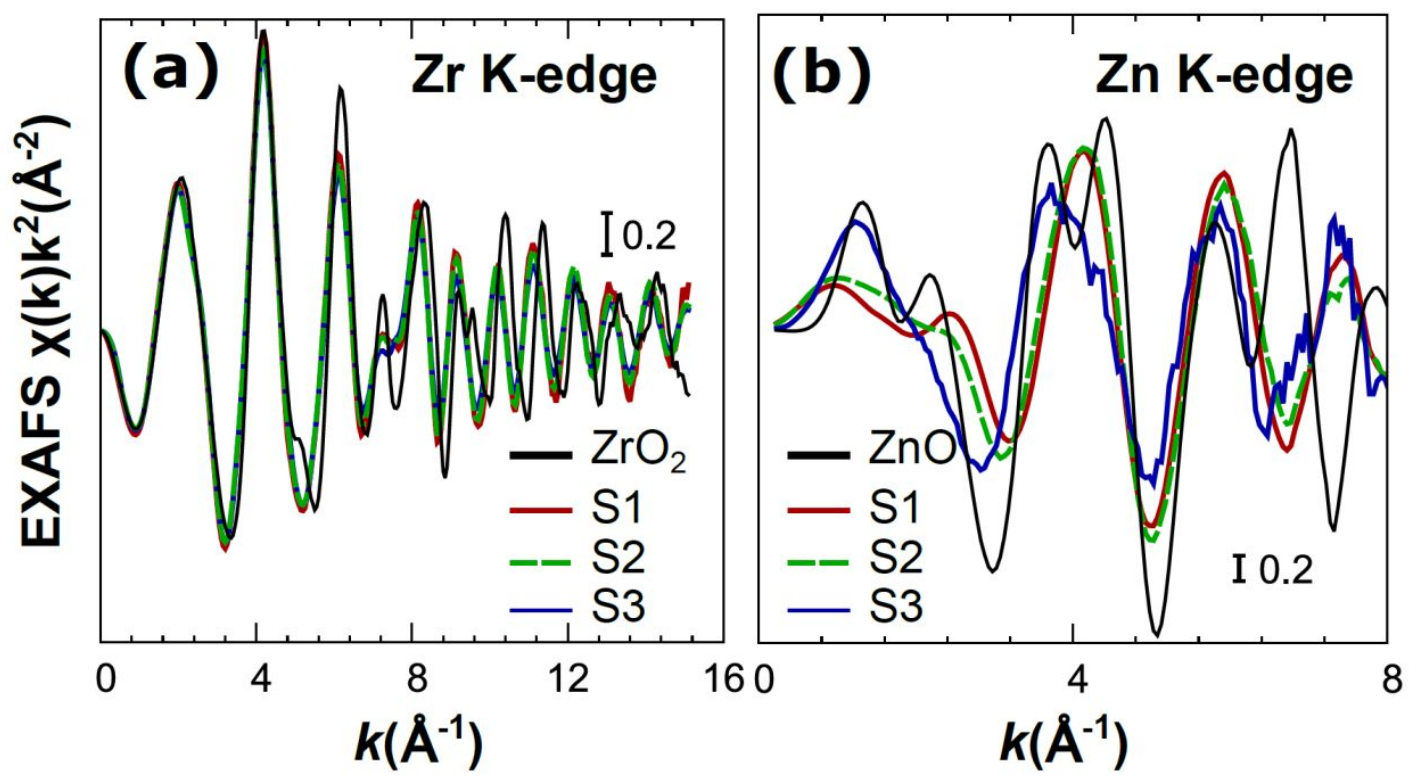

Figure S5 Zr K-edge (a) and Zn K-edge (b) EXAFS spectra for UiO-66 MOF samples S1, S2 and S3, and for reference oxides.

For quantitative EXAFS analysis we performed non-linear least square fitting of EXAFS data. At Zr K-edge, we fit contributions of the first two coordination shells. At Zn K-edge, only the first coordination shell contribution can be reliably analyzed.
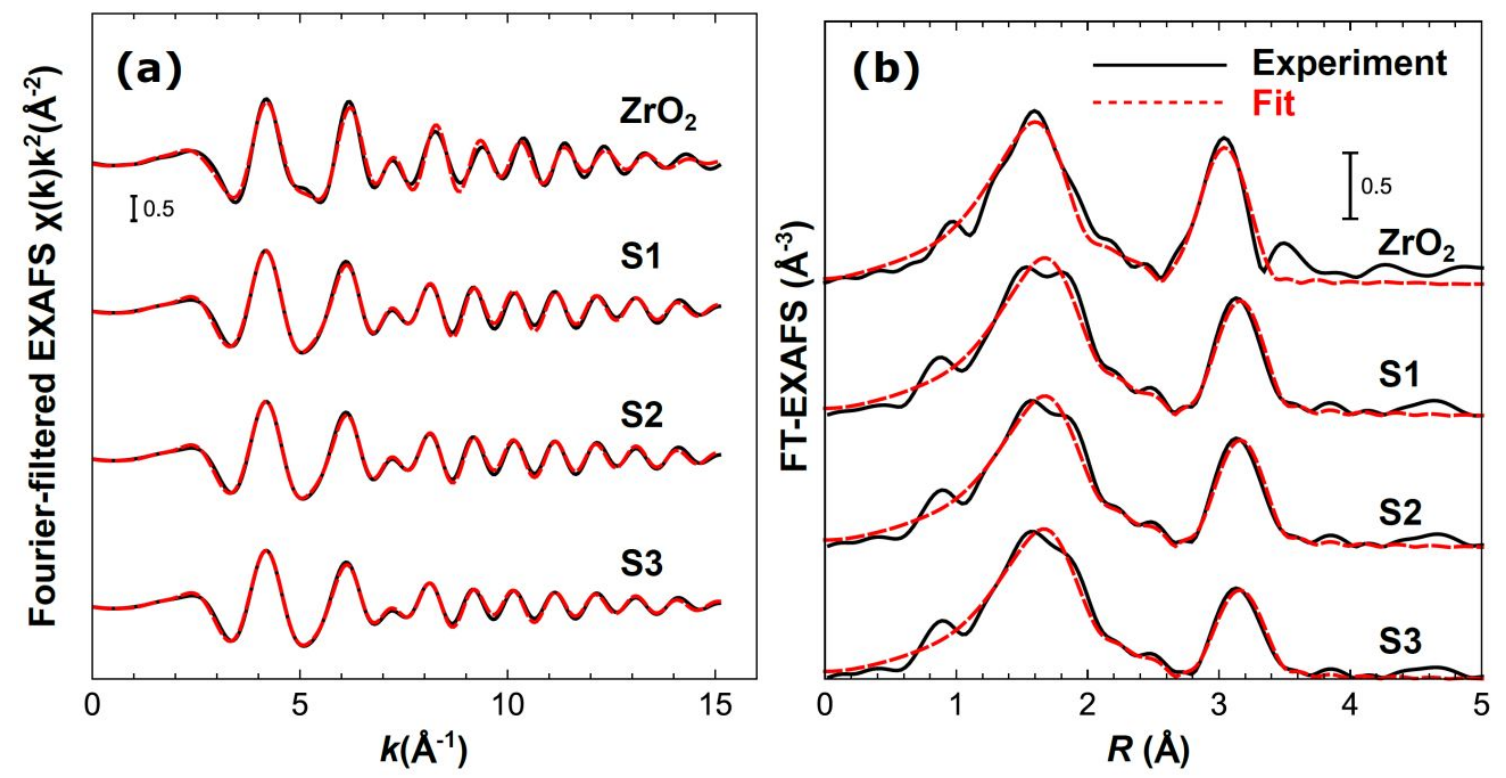

Figure S6 Results of Zr K-edge EXAFS data fitting: comparison of experimental and modeled EXAFS data. Fouriertransformed EXAFS data in R-space, and Fourier-filtered EXAFS data in k-space are shown.

At the Zr K-edge we found that EXAFS spectra can be well reproduced by a combination of contributions of $\mathrm{Zr}-\mathrm{O}$ and $\mathrm{Zr}-\mathrm{Zr}$ bonds. The parameters of $\mathrm{Zr}-\mathrm{O}$ bond is similar to those in $\mathrm{ZrO}_{2}$, but the $\mathrm{Zr}-\mathrm{O}$ is, nevertheless, noticeably larger, suggesting significant difference in the local structure of $\mathrm{Zr}$ in $\mathrm{ZrO}_{2}$ and MOF samples. $\mathrm{Zr}$ $\mathrm{Zr}$ bond length $(3.534 \pm 0.003 \AA)$, in turn, in all samples was significantly larger than $\mathrm{Zr}$ - $\mathrm{Zr}$ distance in $\mathrm{ZrO}_{2}$, and is in an excellent agreement with the $\mathrm{Zr}-\mathrm{Zr}$ distance reported for (Ce, $\mathrm{Zr}$ ) UiO-66 MOFs (3.529 \pm 0.003$){ }^{6}$ 
The MOF-like local environment of $\mathrm{Zr}$ species in all samples is thus confirmed. We note here that the inclusion of Zr-Zn did not improve the fit quality. Thus, Zr K-edge EXAFS data analysis does not confirm directly the presence of mixed $\mathrm{Zr}$ - Zn clusters. However, this is hardly surprising considering that $\mathrm{Zr}$ to $\mathrm{Zn}$ ratio is between 6 and 10 even in the pristine sample (i.e., contribution of Zr-Zn may be too low to be detectable in Zr K-edge EXAFS directly). We can, nevertheless, note that the observed decrease in $\mathrm{Zr}-\mathrm{M}$ metal peak upon decrease in $\mathrm{Zn}$ content is marked by a decrease in $\mathrm{Zr}-\mathrm{M}$ coordination number. This may suggest that some $\mathrm{Zr}-\mathrm{Zn}$ contribution, although masked by much stronger $\mathrm{Zr}-\mathrm{Zr}$ contribution, is present in the $\mathrm{Zr}$ K-edge spectrum of pristine MOF sample, and is removed upon washing treatment.

Zn species, which are minority species in this sample, in principle, should be more sensitive to the presence of $\mathrm{Zn}$-Zr clusters. However, the lack of distant coordination shell contribution in Zn K-edge EXAFS strongly suggests the lack of significant amounts of Zr-Zn clusters. Due to the disordered local environment of $\mathrm{Zn}$, only the $\mathrm{Zn}-\mathrm{O}$ contribution can be analyzed quantitatively. Here we conclude that the $\mathrm{Zn}-\mathrm{O}$ bond is longer in MOF $\mathrm{S} 1$ and $\mathrm{S} 2$ samples than in $\mathrm{ZnO}$ reference and in S3 sample. Moreover, the slightly reduced $\mathrm{Zn}-\mathrm{O}$ coordination number suggests strongly defective local structure for $\mathrm{Zn}$ species in all samples, which gets even more disordered for samples after washing treatment.
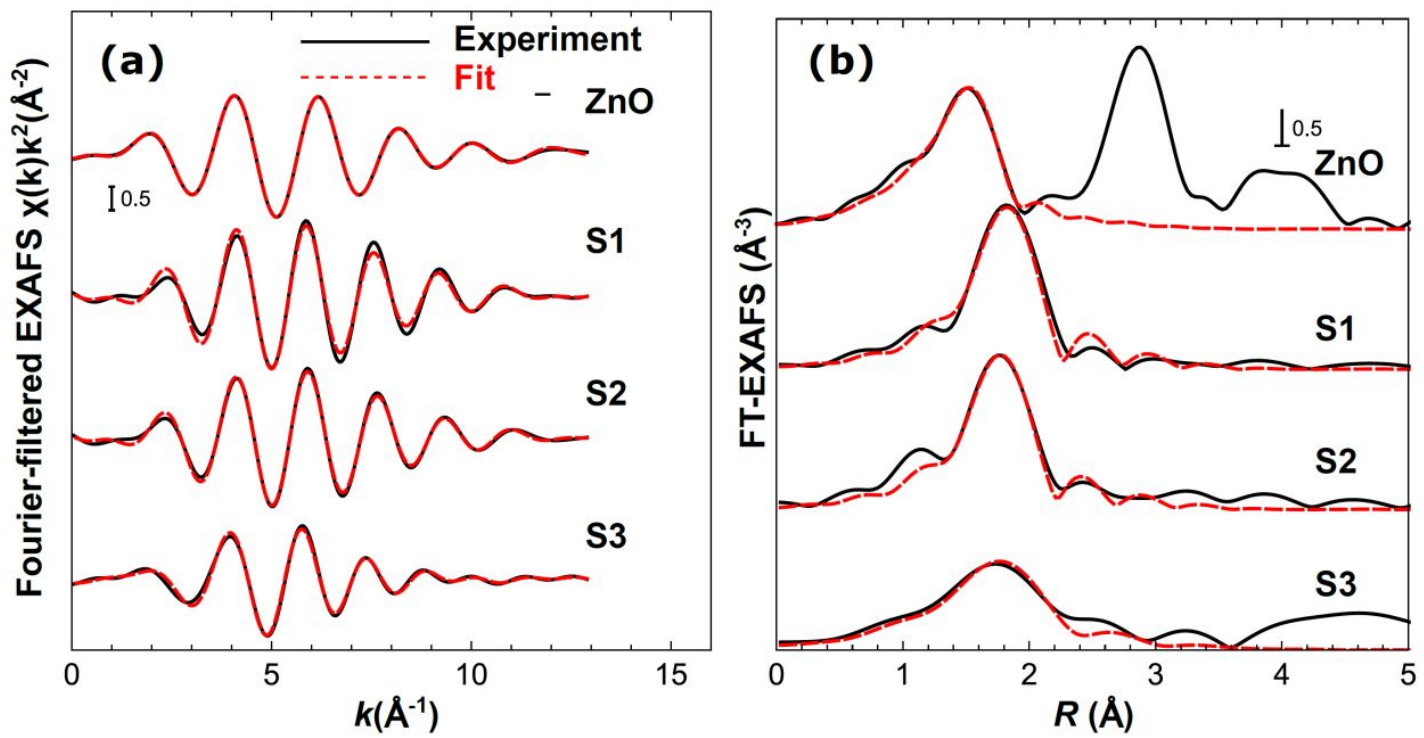

Figure S7. Results of Zn K-edge EXAFS data fitting: comparison of experimental and modeled EXAFS data. Fourier-transformed EXAFS data in $R$-space, and Fourier-filtered EXAFS data in $k$-space are shown. Note that Fourier transform for sample S3 was carried out in shorter $k$-range (up to $k_{\max }=8 \AA^{-1}$ ) than for samples S1 and S2 (up to $k_{\max }=10 \AA^{-1}$ ), which affects the appearance of FT-EXAFS features. 
Table S1. Structure parameters (coordination numbers $N$, interatomic distances $R$, disorder factors $\sigma^{2}$ ), obtained in fitting of experimental $\mathrm{Zr}$ K-edge EXAFS data. Uncertainties of the last digit are given in parentheses.

\begin{tabular}{|c|c|c|c|c|c|c|c|c|}
\hline Sample & $N_{Z r-o}$ & $R_{Z r-o}(\AA ̊)$ & $\mid \sigma_{Z r-o}^{2}\left(\AA^{2}\right)$ & $N_{Z r-Z r}$ & $R_{Z r-Z r}(\AA ̊)$ & $\mid \begin{array}{l}\sigma_{Z r-Z r}^{2} \\
\left(\AA^{2}\right)\end{array}$ & $\Delta E_{0}(\mathrm{eV})$ & $R$ factor \\
\hline $\mathrm{ZrO}_{2}$ & $6.0(9)$ & $2.15(1)$ & $0.011(2)$ & $5(1)$ & $3.460(9)$ & $0.008(1)$ & $-10(1)$ & $4.2 \%$ \\
\hline S1 & $6.7(7)$ & $2.22(1)$ & $0.013(2)$ & $2.5(5)$ & $3.534(6)$ & $0.004(1)$ & $-4(1)$ & $1.7 \%$ \\
\hline S2 & $6.3(6)$ & $2.22(1)$ & $0.013(2)$ & $2.2(4)$ & $3.535(5)$ & $0.004(1)$ & $-4(1)$ & $1.4 \%$ \\
\hline S3 & $6.2(5)$ & $2.21(1)$ & $0.013(71)$ & $1.9(4)$ & $3.534(7)$ & $0.004(1)$ & $-4(1)$ & $1.3 \%$ \\
\hline
\end{tabular}

Table S2. Structure parameters (coordination numbers $N$, interatomic distances $R$, disorder factors $\boldsymbol{\sigma}^{2}$ ), obtained in fitting of experimental Zn K-edge EXAFS data. Uncertainties of the last digit are given in parentheses.

\begin{tabular}{l|l|l|l|l|l} 
Sample & \multicolumn{1}{|c|}{$\boldsymbol{N}_{\boldsymbol{Z n}-\boldsymbol{o}}$} & $\boldsymbol{R}_{\boldsymbol{Z n}-\boldsymbol{o}(\AA)}$ & $\boldsymbol{\sigma}_{\boldsymbol{Z n}-\boldsymbol{o}}^{2}\left(\AA^{2}\right)$ & $\boldsymbol{\Delta} \boldsymbol{E}_{\mathbf{0}}(\mathrm{eV})$ & $R$ factor \\
\hline $\mathrm{ZnO}$ & $4.0(2)$ & $1.94(2)$ & $0.006(8)$ & $-6(2)$ & $0.2 \%$ \\
\hline $\mathrm{S} 1$ & $3(1)$ & $2.09(9)$ & $0.001(5)$ & $9(6)$ & $2.6 \%$ \\
$\mathrm{~S} 2$ & $2.8(4)$ & $2.08(5)$ & $0.001(2)$ & $8(4)$ & $1.0 \%$ \\
$\mathrm{~S} 3$ & $2.2(7)$ & $1.9(1)$ & $0.001(4)$ & $-8(9)$ & $1.6 \%$ \\
\hline
\end{tabular}

\section{Reference}

1. Shearer, G. C.; Chavan, S.; Ethiraj, J.; Vitillo, J. G.; Svelle, S.; Olsbye, U.; Lamberti, C.; Bordiga, S.; Lillerud, K. P., Tuned to Perfection: Ironing Out the Defects in Metal-Organic Framework UiO-66. Chemistry of Materials 2014, 26 (14), 4068-4071.

2. Schenk, T.; Anspoks, A.; Jonane, I.; Ignatans, R.; Johnson, B. S.; Jones, J. L.; Tallarida, M.; Marini, C.; Simonelli, L.; Hönicke, P.; Richter, C.; Mikolajick, T.; Schroeder, U., Local structural investigation of hafnia-zirconia polymorphs in powders and thin films by X-ray absorption spectroscopy. Acta Mater. 2019, 180, 158-169.

3. Ravel, B.; Newville, M., ATHENA, ARTEMIS, HEPHAESTUS: data analysis for X-ray absorption spectroscopy using IFEFFIT. Journal of Synchrotron Radiation 2005, 12 (4), 537-541.

4. Ankudinov, A. L.; Conradson, S. D.; Mustre de Leon, J.; Rehr, J. J., Relativistic XANES calculations of Pu hydrates. Physical Review B 1998, 57 (13), 7518-7525.

5. Øien, S.; Wragg, D.; Reinsch, H.; Svelle, S.; Bordiga, S.; Lamberti, C.; Lillerud, K. P., Detailed Structure Analysis of Atomic Positions and Defects in Zirconium Metal-Organic Frameworks. Cryst Growth Des 2014, 14 (11), 5370-5372.

6. Lomachenko, K. A.; Jacobsen, J.; Bugaev, A. L.; Atzori, C.; Bonino, F.; Bordiga, S.; Stock, N.; Lamberti, C., Exact Stoichiometry of CexZr6-x Cornerstones in Mixed-Metal UiO-66 Metal-Organic Frameworks Revealed by Extended Xray Absorption Fine Structure Spectroscopy. J. Am. Chem. Soc. 2018, 140 (50), 17379-17383. 